\title{
Development and Analysis of New NLFM Waveform for Achieving Sidelobe Reduction and Narrowing of Main lobe Width
}

\author{
Kavitha Chandu * \\ Department of Physics, GITAM Institute of Science \\ GITAM Deemed to be University \\ Visakhapatnam, 530045, A.P, India \\ *kchandu@gitam.edu
}

\begin{abstract}
Aimed at narrowing main lobe width and reduced sidelobe values, we developed three new NLFM chirp waveforms. The ambiguity function and the impact of sampling rate and compression ratios of these waveforms are analyzed. Their performance is examined against the doppler effect and background noise. One of the three designed NLFM chirp waveforms is useful in applications requiring side lobes of $50 \mathrm{~dB}$ and narrow main lobe width. The new waveform could achieve reduced sidelobes and narrow main lobe width compared to LFM and other NLFM waveforms.
\end{abstract}

Key words: LFM, NLFM, Signal to Noise Ratio, Peak SideLobe Level

\section{INTRODUCTION}

Pulse compression is used in radar systems for range resolution and long-range detection since 1950s. Radars have been transformed from analog to digital from 1960s improving pulse compression. Digital radars can perform pulse compression using various techniques. Among the pulse compression techniques, LFM is the most popular technique because of its ease of generation and doppler tolerance [1]. The main drawback of LFM, however, is yielding high sidelobe levels. As such, it cannot detect weak signals. Methods such as windowing, filtering, and optimization are applied to reduce sidelobe levels. Alternatively, NLFM is developed in 2009 to overcome the drawbacks of LFM [2]. Most of the studies focused on NLFM chirp waveform design. The chirp modulation can shape power spectral density such that autocorrelation function yields reduced side lobes at the cost of main lobe broadening, which can diminish target detection accuracy. The main drawback of NLFM signals is that several filters are required at the receiver to detect target return because these signals are doppler intolerant. Signal distortion caused by Doppler shift and background noise is another drawback of NLFM signals. In radar systems [3], noise cannot be avoided, especially the additive white gaussian noise that is naturally present in all the devices. A moving target causes the Doppler effect, which affects the performance of pulse compression. Although several studies have attempted at examining this problem, sidelobe reduction and narrowing of main lobe width continue to be attracting interest of researchers [4-11].

The present study focuses on new chirp waveforms to preserve the accuracy and to obtain reduced side lobes. The effect of sampling rate and compression ratio are studied, and the ambiguity function is plotted and analyzed for doppler tolerance, sidelobe performance, distance, and doppler frequency coupling. The chirp waveforms are investigated for Doppler shift and background noise.

\section{RADAR PULSE COMPRESSION}

\subsection{Linear Frequency Modulation (LFM)}

LFM is the most commonly used technique in radar applications because of its good resolution, doppler tolerance, and easy generation. The first sidelobe level of LFM is around $-13.3 \mathrm{~dB}$. The chirp waveform of LFM is linear and represented mathematically as

$\mathrm{s}(\mathrm{t})_{\mathrm{LFM}}=\exp (\mathrm{j} \Phi(\mathrm{t})),-\tau / 2 \leq \mathrm{t} \leq \mathrm{t} / 2$

\subsection{Nonlinear Frequency Modulation (NLFM)}

Another type of pulse compression technique is NLFM. It is a non-linear FM waveform in which the relationship between frequency and signal time is not linear. It has better range resolution, lower sidelobe values, and higher SNR. The most popular NLFM chirps are cosine spectrum shape, tangent based and truncated Gaussian waveforms. The NLFM chirp waveform is

$$
\mathrm{s}(\mathrm{t})_{\mathrm{NLFM}}=\exp (\mathrm{j} \Phi(\mathrm{t}))
$$


where $\Phi(t)$, the instantaneous frequency obtained using the differential of phase modulation. In this study, the following cosine-based chirp waveforms have been developed.

$$
\begin{aligned}
& \left(\cos (\mathrm{k} 1 * \mathrm{t} / \mathrm{tau})^{\mathrm{k} 2}\right) / \mathrm{k} 3 \\
& \mathrm{k} 4 *\left(\cos (\mathrm{k} 1 * \mathrm{t} / \mathrm{tau})^{\mathrm{k} 2}\right)- \\
& \mathrm{k} 4 *\left(\cos (\mathrm{k} 1 * \mathrm{t} / \mathrm{tau})^{\mathrm{k} 2}\right) / \mathrm{k} 3 \text {------ NLFM III }
\end{aligned}
$$

where $\mathrm{k} 1, \mathrm{k} 2, \mathrm{k} 3$ and $\mathrm{k} 4$ are constants.

\section{PERFORMANCE ANALYSIS}

\subsection{Main lobe width and peak sidelobe level}

The performance of resolution and detectability in radar systems is related to main lobe width and peak sidelobe level. Simulations are carried out for different compression ratios $(\beta \tau)$ and sampling rates(fs) to analyze and compare the newly designed chirp waveforms. The results are tabulated in Tables 1 and 2. It may be noticed that for $\beta \tau=400$ and fs $=5 \beta$, the three chirp waveforms yielded the lowest sidelobe levels of $-60.29,-62.16$, and $-56.69 \mathrm{~dB}$, respectively. However, the main lobe width broadened compared to LFM. For other compression ratios $\beta \tau=48,32,100$ and sampling rates of $\mathrm{fs}=5 \beta$ and $10 \beta$, the peak sidelobe level of all the designed waveforms varied between $-47.4 \mathrm{~dB}$ and -55.46 $\mathrm{dB}$. For a sampling rate of $10 \beta$ and compression ratios of 32 and 100, NLFM III achieved narrow main lobe width and lower sidelobe levels than LFM. At the same time, other NLFM waveforms (NLFM I and NLFM II) exhibited broader main lobe width and reduced sidelobe levels. Figure 1. depicts the matched filter response of LFM and NLFM waveforms for $\beta \tau=100$ and $f s=10 \beta$. The figure shows that the first sidelobe level of NLFM waveforms has a lower sidelobe level by $37.85 \mathrm{~dB}$ than LFM. NLFM III yielded narrow main lobe width (less than LFM) with reduced side lobes among the three waveforms. Hence the analysis is done using this waveform.

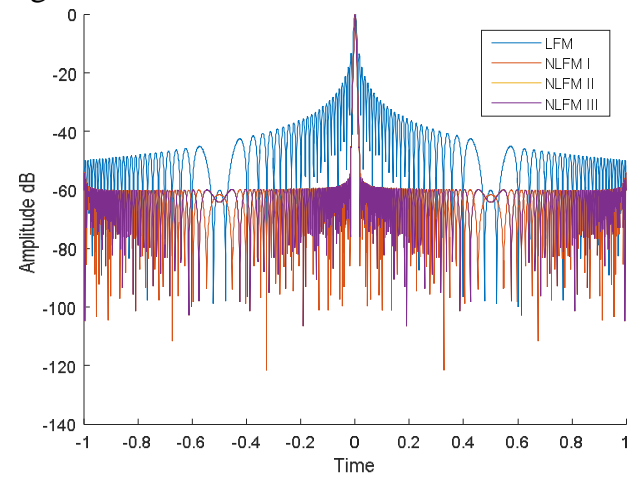

Figure 1. Matched filter response of LFM and NLFM signals
Table 1. Peak sidelobe levels of LFM and new NLFM signals

\begin{tabular}{|c|c|c|c|c|c|}
\hline $\begin{array}{c}\text { Compression } \\
\text { Ratio }\end{array}$ & $\begin{array}{c}\text { Sampling } \\
\text { Rate }\end{array}$ & \multicolumn{4}{|c|}{$\begin{array}{c}\text { Main lobe width } \\
-\mathbf{3 ~ d B}\end{array}$} \\
\hline & & LFM & $\begin{array}{l}\text { NLFM } \\
\text { I }\end{array}$ & $\begin{array}{l}\text { NLFM } \\
\text { II }\end{array}$ & $\begin{array}{l}\text { NLFM } \\
\text { III }\end{array}$ \\
\hline 48 & $5 \beta$ & 0.38867 & 0.80664 & 0.75195 & 0.37988 \\
\hline 400 & $5 \beta$ & 0.39856 & 0.7832 & 0.7511 & 0.40247 \\
\hline 32 & $10 \beta$ & 0.19189 & 0.31494 & 1.0391 & 0.18115 \\
\hline 100 & $10 \beta$ & 0.20117 & 0.32495 & 0.33936 & 0.18921 \\
\hline
\end{tabular}

\begin{tabular}{|l|l|c|c|c|c|}
\hline $\begin{array}{l}\text { Compression } \\
\text { Ratio } \\
\beta \tau\end{array}$ & $\begin{array}{l}\text { Sampling } \\
\text { Rate } \\
\text { Fs }\end{array}$ & \multicolumn{4}{|c|}{$\begin{array}{c}\text { First sidelobe level } \\
\text { dB }\end{array}$} \\
\hline & & LFM & $\begin{array}{c}\text { NLFM } \\
\text { I }\end{array}$ & $\begin{array}{c}\text { NLFM } \\
\text { II }\end{array}$ & $\begin{array}{c}\text { NLFM } \\
\text { III }\end{array}$ \\
\hline 48 & $5 \beta$ & -13.71 & -47.42 & -48.56 & -47.27 \\
\hline 400 & $5 \beta$ & -13.34 & -60.29 & -62.16 & -56.69 \\
\hline 32 & $10 \beta$ & -13.68 & -47.31 & -47.13 & -47.1 \\
\hline 100 & $10 \beta$ & -13.49 & -54.39 & -55.46 & -51.34 \\
\hline
\end{tabular}

Table 2. $3 \mathrm{~dB}$ main lobe width of LFM and NLFM signals

\section{SAMPLING RATE}

The change of the first sidelobe level with the sampling rate for NLFM III is shown in figure 2. It is evident from the figure that the first sidelobe level reduced down to $-50 \mathrm{~dB}$ for $\mathrm{fs}>3 \beta$. As the system performance is affected by the sampling rate, the designed signal has an advantage compared to other NLFM signals in the literature.

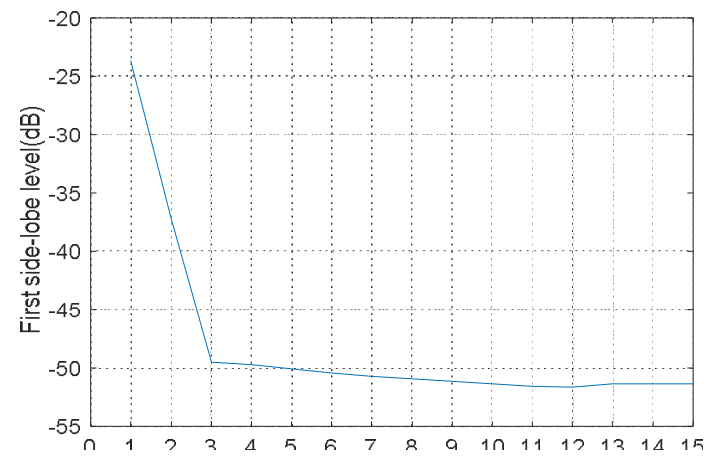

Figure 2. First sidelobe level versus sampling rate

\section{SIGNAL TO NOISE RATIO}

The performance of signal against background noise is investigated (Figure 3.). Simulation is carried out by adding additive white gaussian noise on complex NLFM signals because it is present in all the devices. SNR varied from 20 dB to $-20 \mathrm{~dB}$. 25 trials were carried on at each point of SNR 
and the mean value is noted. The decreased SNR causes the PSL to increase. At $20 \mathrm{~dB}$, the peak sidelobe level of NLFM III is around $-42.72 \mathrm{~dB}$, whereas, for $-20 \mathrm{~dB}$, it is $-19.38 \mathrm{~dB}$.

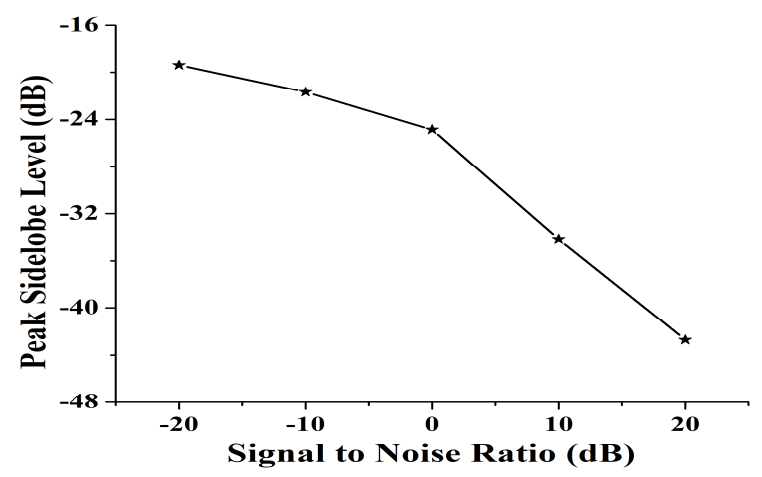

Figure 3. Background noise to PSL of NLFM III

\section{DOPPLER SHIFT}

The chirp signal of NLFM III is examined for Doppler shift corresponding to four different frequencies $\mathrm{f}_{\mathrm{d}}=1,3,5$, and 7. (Figures 4). To measure the Doppler tolerance, the calculated Doppler shift frequency $\left(\mathrm{f}_{\mathrm{d}}\right)$ is added to the center frequency $\left(f_{c}\right)$ of matched filter $\left(\left(f_{c} \pm f_{d}\right)\right)$. From the figures, it can be seen clearly that the matched filter output of designed NLFM signals is distorted which increased with doppler frequency. This degrades the detectability of radar systems. The NLFM waveforms developed in this study, like other waveforms, exhibited doppler intolerance.

\section{AMBIGUITY FUNCTION}

Ambiguity function is an important tool to analyze resolution and detection of moving targets, sidelobe performance, distance, and doppler frequency coupling. It is the absolute value of the output of the matched filter. The filter's input is a doppler shifted version of return signal to which the filter is matched. The function is given by equation

$$
\left|A\left(\tau, f_{d}\right)\right|=\left|\int_{-\infty}^{\infty} s(t) s^{*}(t+\tau) \exp \left(j 2 \pi f_{d} t\right) d t\right|
$$

From Figures $5-8$, It is evident that it is a pin type ambiguity function of new NLFM signals and they have good range resolution. They exhibited better doppler tolerance compared to other NLFM signals. The time and doppler frequency coupling are weaker, which can be seen in the contour maps, The new NLFM signals have clean sidelobes (Zero Doppler curve) compared to LFM but exhibited the same zero delay curve as LFM.
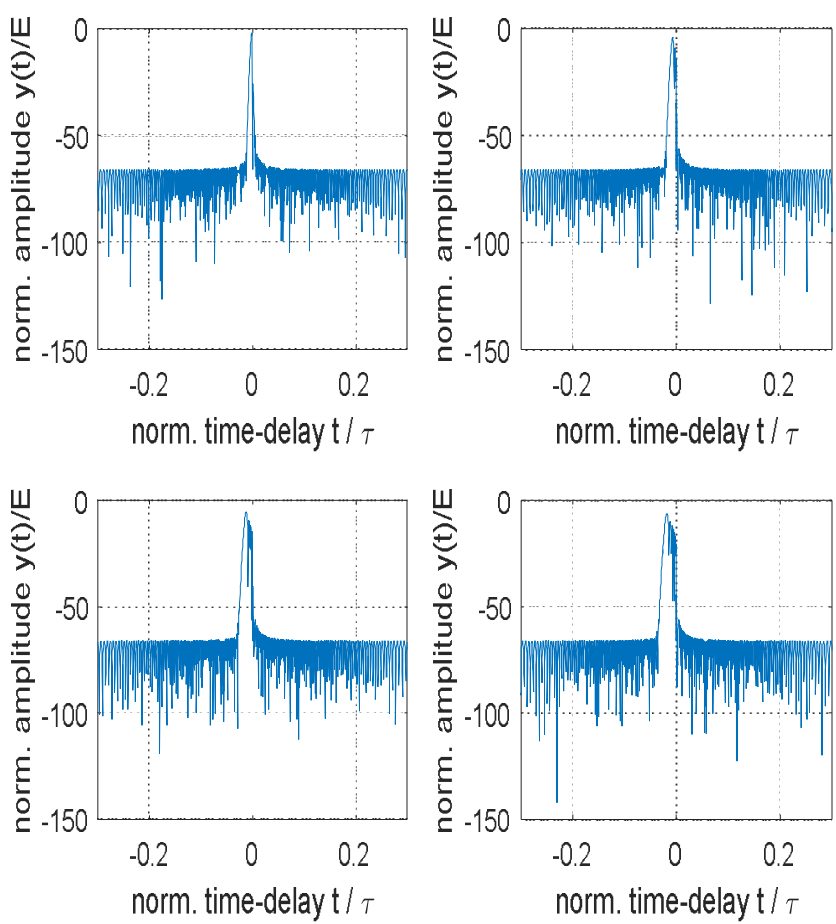

Figure 4. Matched filter output response of NLFM III when doppler shift is $1 / \tau, 3 \tau, 5 / \tau, 7 / \tau$
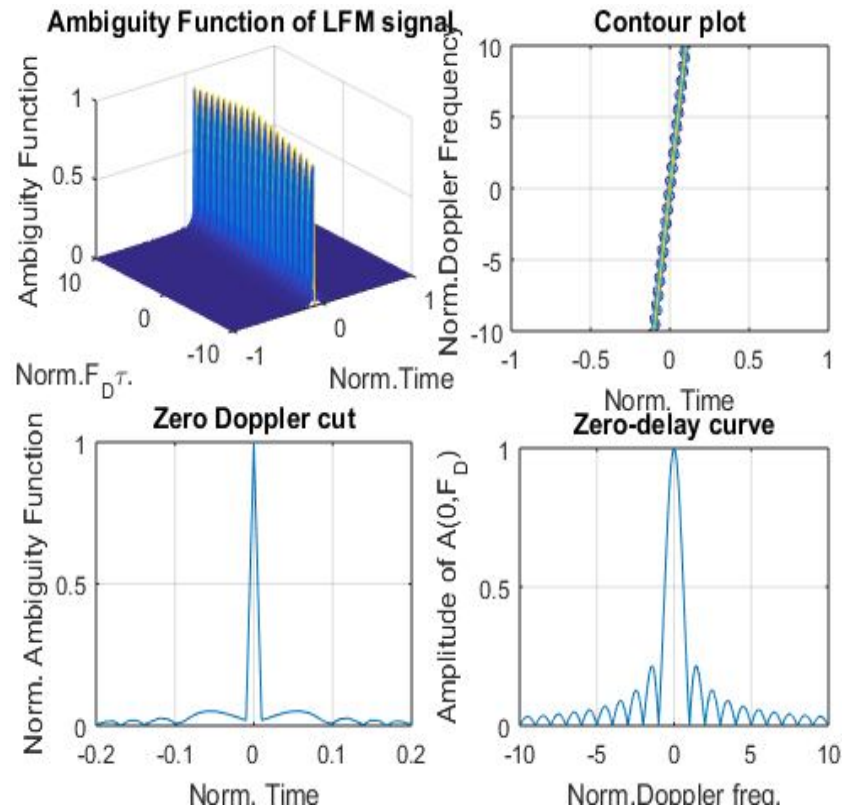

Figure 5. Ambiguity function, contour map, zero-Doppler cut and zero-Delay curve of LFM signal 


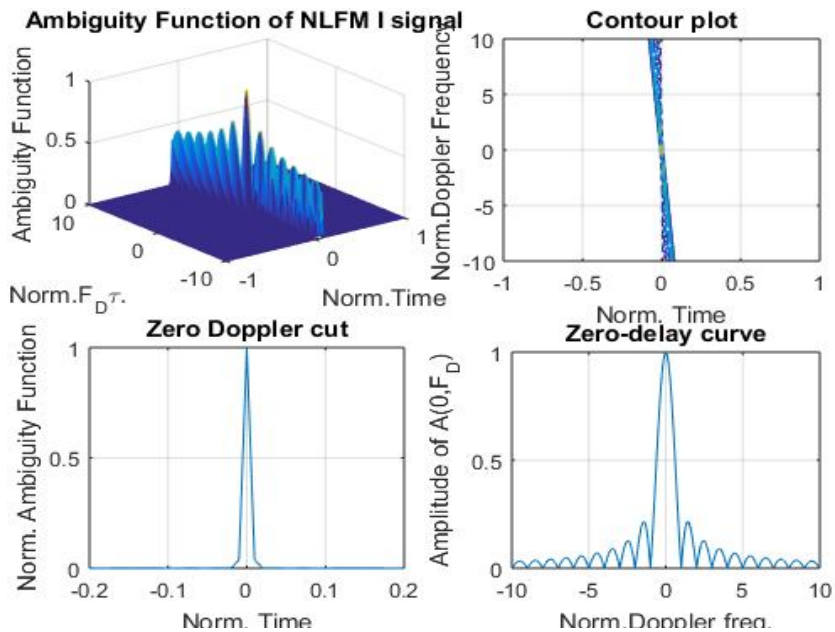

Figure 6. Ambiguity function, contour map, zero-Doppler cut and zero-Delay curve of NLFM I signal
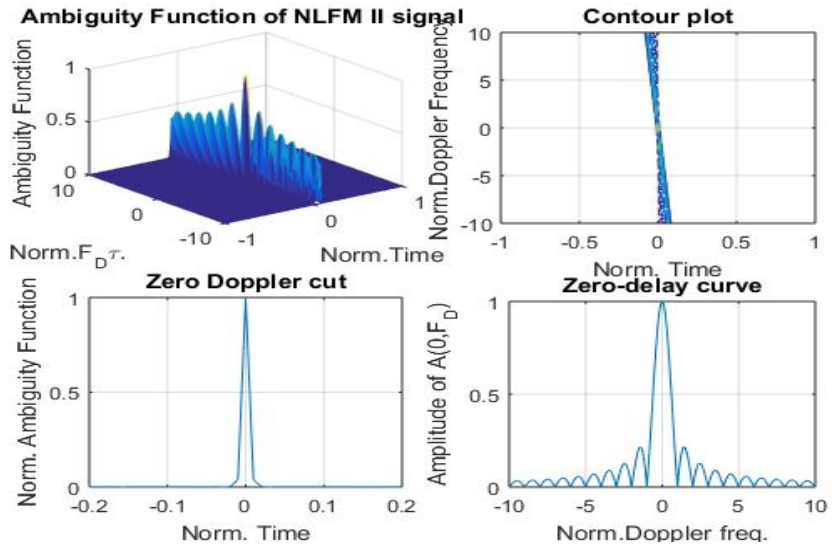

Figure 7. Ambiguity function, contour map, zero-Doppler cut and zero-Delay curve of NLFM II signal
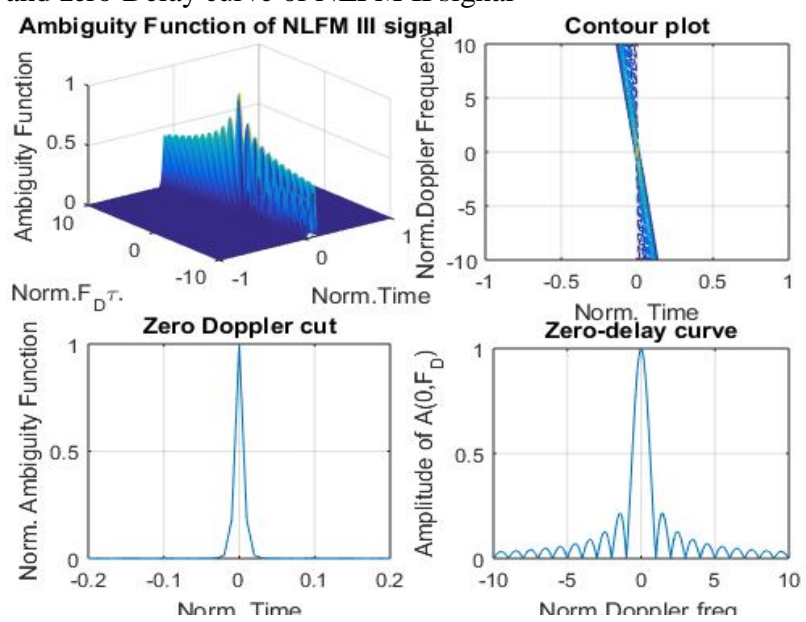

Figure 8. Ambiguity function, contour map, zero-Doppler cut and zero-Delay curve of NLFM III signal

\section{CONCLUSION}

In this study, a new NLFM waveform useful for radar applications that require sidelobes of $-50 \mathrm{~dB}$ and narrow main lobe width is designed and analyzed. The signal energy of the NLFM III waveform is conserved due to the subtly widened main lobe. The $3 \mathrm{~dB}$ main lobe width at the sampling rate of $10 \beta$ and for $\beta \tau=100$ is 0.18921 . The peak sidelobe level is $-51.34 \mathrm{~dB}$. Hence, it may be inferred that NLFM III could achieve reduced sidelobes along with narrowing main lobe width compared to LFM and other NLFM waveforms. NLFM I and NLFM II achieved a peak sidelobe level of about $-60 \mathrm{~dB}$ at a $5 \beta$ sampling rate at the cost of widened main lobe. The new NLFM signals exhibited clean sidelobe levels. The same zero-delay curve as that of LFM and doppler tolerance compared to other NLFM signals in literature is also observed. The PSL value of the NLFM waveforms reduced with the increased signalto-noise ratio. However, the compressed signal is still distorted by the Doppler shift like other drawbacks of NLFM methods.

\section{ACKNOWLEDGEMENT}

This research did not receive any specific grant from funding agencies in the public, commercial, or not-for-profit sector.

\section{CONFLICT OF INTEREST}

Single author

\section{REFERENCES}

1. Michael Picciolo, et al. "Adaptive LFM waveform diversity", Radar Conference 2008, RADAR'08. IEEE, 2008. DOI: 10.1109/RADAR.2008.4721067

2. Yee Kit Chan et al. "Side lobes reduction using simple two and tri-stages nonlinear frequency modulation (NLFM)", Progress In Electromagnetics Research, vol. 98, pp. 33-52, 2009. doi:10.2528/PIER09073004

3. N. Adithyavalli et al. "Modified Radar Signal Model using NLFM", International Journal of Recent Technology and Engineering (IJRTE), Volume-8 (2S3), pp. 513-516, July 2019. DOI: 10.35940/ijrte.B1091.0782S319

4. Khanh NV, Turley MDE. "A range sidelobe suppression technique based on adaptive spectral shaping for LFM waveforms". In: 2018 International Conference on Radar (RADAR), Brisbane, QLD. 2018, pp. 1-6. DOI: 10.1109/RADAR.2018.8557251

5. Saeedi J, Faez K. "Synthetic aperture radar imaging using nonlinear frequency modulation signal”. IEEE Transactions on Aerospace and Electronic Systems, 52(1), pp.99-110, 2016. Available from: https://dx.doi.org/10.1109/taes.2015.140310

6. Vizitiu IC, et al. "Sidelobe reduction in pulsecompression radar using the stationary phase technique: An extended comparative study". In: 2014 International Conference on Optimization of Electrical 
and Electronic Equipment (OPTIM), Bran, pp.898901,2014. DOI: 10.1109/OPTIM.2014.6850932

7. YueWenzhen, Zhang Yan, "A novel nonlinear frequency modulation waveform design aimed at side-lobe reduction", Signal Processing Communications and Computing (ICSPCC) 2014 IEEE International Conference on, 2014. DOI: 10.1109/ICSPCC.2014.6986266

8. Jun Song et al., "Analysis and Detection of S-Shaped NLFM Signal Based on Instantaneous Frequency", Journal of Communications, vol. 10(12), 2015. DOI: $10.12720 /$ jcm.10.12.976-982

9. Budiman P.A. et al., "Side lobe suppression on pulse compression using curve-shaped nonlinear frequency modulation", 1st International conference on Information Technology Information Systems and
Electrical Engineering (ICITISEE) 2016. DOI: 10.1109/ICITISEE.2016.7803046

10. Kavitha Chandu et al., "Side-Lobe Reduction Using Polynomial Based NLFM Function", International Journal of Advanced Science and Technology, Vol. 29(7), $\quad$ pp. $11843 \quad-\quad 1184, \quad 2020$. http://sersc.org/journals/index.php/IJAST/article/view/2 7858

11. Kavitha C, Valli NA, Dasari M. "Optimization of twostage NLFM signal using Heuristic approach”, Indian Journal of Science and Technology 13(44), pp.44654473,2020. https://doi.org/10.17485/IJST/v13i44.1841 